\title{
Identification of a Patient Population Previously Not Considered for Organ Donation
}

\author{
Brad Barrois 1 \\ 1. Quality, Louisiana Organ Procurement Agency \\ $\square$ Corresponding author: Brad Barrois, bbarrois@lopa.org \\ Disclosures can be found in Additional Information at the end of the article
}

\section{Abstract}

For the foreseeable future, more individuals will need a kidney than there are kidneys available for transplant. This is not a new issue, and it is one that will not likely be solved anytime soon. While recent initiatives have focused on efficiently allocating kidneys in order to maximize supply, a shortage will remain.

Currently, organs are made available for transplant through three different processes: donation after brain death declaration (BD), donation after circulatory death (DCD), and living donation (one healthy individual donates to a person in need). The objective of this article is to discuss the possibility of a fourth option in imminent death single kidney donation (IDSKD) and its potential effects on the future of donation and transplantation. During our study, IDSKD had the potential to increase the number of kidneys transplanted in our service area by approximately $5 \%$.

Categories: Transplantation

Keywords: organ donation, kidney transplant

\section{Introduction}

The purpose of this study was to identify an alternative group of patients who were unable to become an organ donor after brain death declaration or donation after circulatory death. The primary reason these patients were unable to donate is due to intact neurological responses, which typically would prevent the patient from cardiac arresting within a given timeframe. All patients who were focused on during the study were referred to the Louisiana Organ Procurement Agency (LOPA) due to being hospital-bound, ventilator dependent, and their families had made the decision to terminally extubate.

Received 07/29/2016 Review began 08/01/2016 Review ended 09/20/2016 Published 09/26/2016

C) Copyright 2016

Barrois. This is an open access article distributed under the terms of the Creative Commons Attribution License CC-BY 3.0., which permits unrestricted use, distribution, and reproduction in any medium, provided the original author and source are credited.
Imminent death single kidney donation (IDSKD) would allow a patient population who currently are deemed unsuitable for organ donation to become a donor. Once the patient's family has decided to terminally extubate, the patient would be evaluated for donation after circulatory death (DCD). If the organ procurement organization (OPO) determines that DCD is not an option, then the patient could be evaluated for IDSKD. This process allows a family to authorize the removal of a single kidney from the potential organ donor. If the family approves of this option, the OPO and hospital would complete all legally required paperwork and required testing, per the United Network for Organ Sharing (UNOS) policy. Once a recipient is identified, the donor would be brought to the operating room, and a nephrectomy would be performed by the recipient's transplant surgeon. Once the surgery is complete, the donor would 
then be brought back to the ICU, or designated area. The family's decision to terminally extubate the patient could then be carried out by the appropriate hospital staff.

While this process is not an original thought, addressing the issue of an eligible patient population is. To date, no known long-term study has been completed to assess the potential for this type of organ donation.

\section{Materials And Methods}

This study focused on data collected from 2014-2015. The original use of the data used in this study was not intended for this project. This study is a byproduct of research currently being completed by the Louisiana Organ Procurement Agency (LOPA) attempting to accurately predict the likelihood a patient would cardiac arrest in a timely manner in order to facilitate DCD. In order for donation after circulatory death to take place, the potential donor must cardiac arrest within two hours. Outside of the allotted time, the potential donor is no longer eligible for organ donation. The original research evaluated numerous patient demographics including, but not limited to, age, body mass index (BMI), cause of death, intact neurological responses, and various lab values. Patients would be evaluated once the LOPA was informed of a family's decision to terminally extubate a patient who was ventilator dependent and hospitalbound. The LOPA clinical staff were responsible for the initial data collection and evaluation.

Under the advisement of the LOPA's medical director, additional metrics would be necessary to properly assess the potential donor pool for IDSKD, primarily a kidney donor profile index (KDPI), history of diabetes, and the patient's age. A patient's KDPI is determined using the KDPI calculator provided by the Organ Procurement and Transplantation Network (OPTN). Variables used in the calculation are as follows: age, height, weight, ethnicity, history of hypertension, history of diabetes, cause of death, serum creatinine, as well as Hepatitis C status. Once all information is entered, the calculator provides a percentage. According to OPTN, "lower KDPI score is associated with longer estimated function, while higher KDPI scores are associated with shorter estimated function. For example, a kidney with a KDPI of $20 \%$ is expected to have shorter longevity than $20 \%$ of recovered kidneys (i.e., longer function than $80 \%$ of recovered kidneys)" [1]. Figure 1 compares estimated graft half-lives (years) of a living donor to a deceased donor as the KDPI increases [1].

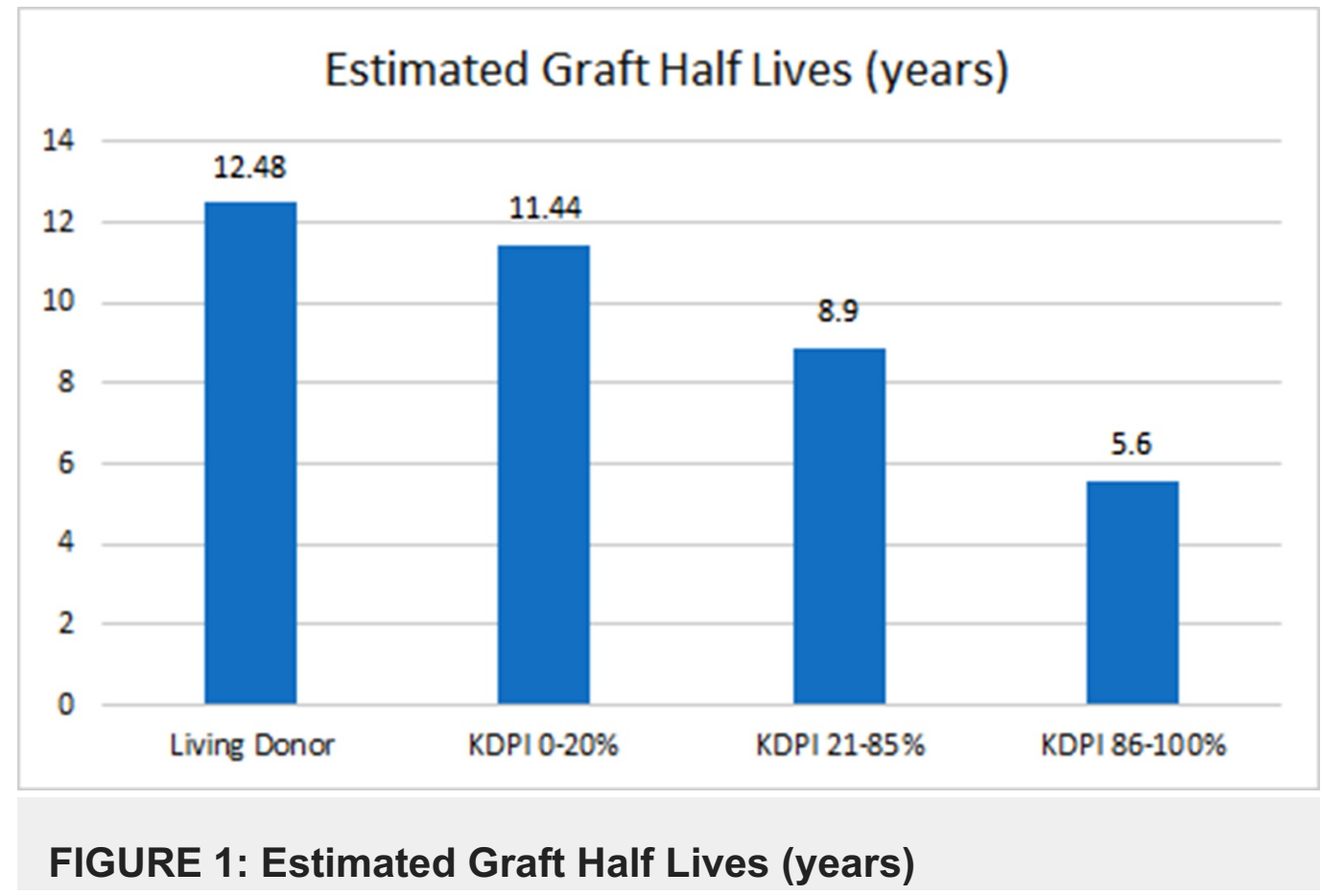


Since IDSKD is neither a deceased donor nor is it a traditional living donor, a metric, such as a KDPI, was needed for evaluation. Given that it provides an estimation of graft function, it was determined to be a pertinent metric for evaluating the donor population for IDSKD.

Traditionally, a potential donor's KDPI is only calculated in a deceased donation scenario, not for living donors. In imminent death single kidney donation, the patient does not fit the definition for either type of donation, making the use of KDPI unique for the current study.

Calculation of the potential donor's KDPI was completed by the LOPA's Data Manager. All necessary data needed for completion of the KDPI calculation were obtained after a potential donor was referred to the LOPA.

Once data collection was completed, it then needed to be filtered according to the LOPA's medical director's criteria. The following criteria were used as filters: patients over the age of 60 were removed, those patients that had become a donor, those declared brain dead, history of diabetes, a KDPI above $85 \%$, or if the family authorized for donation but the process was stopped due positive serological results, poor organ function, or inability to locate a potential recipient.

\section{Results}

Twenty-five potential imminent death single kidney donors were identified. These patients' ages ranged from 14 to 59 years of age, with a median age of 45 and mean of 44 . Calculated KDPI's ranged from $15 \%$ to $84 \%$, with a median of $59 \%$ and a mean of $56 \%$.

Table 1 displays the identified potential donors with their corresponding KDPI as well as age. The list is sorted by descending age. 


\section{Cureus}

\begin{tabular}{|c|c|c|}
\hline Case \# & KDPI & Age \\
\hline 14812180513 & $84 \%$ & 59 \\
\hline 15127212817 & $83 \%$ & 59 \\
\hline 14504174134 & $70 \%$ & 56 \\
\hline 14606125758 & $63 \%$ & 56 \\
\hline 14718110423 & $83 \%$ & 56 \\
\hline 14728081000 & $73 \%$ & 55 \\
\hline 14322042142 & $70 \%$ & 54 \\
\hline 14402082732 & $52 \%$ & 54 \\
\hline 14902033139 & $59 \%$ & 52 \\
\hline 14318010316 & $30 \%$ & 50 \\
\hline 14819060522 & $57 \%$ & 46 \\
\hline 15116151900 & $82 \%$ & 46 \\
\hline 14409004803 & $45 \%$ & 45 \\
\hline 14715102508 & $45 \%$ & 44 \\
\hline 15222000521 & $55 \%$ & 43 \\
\hline 14501125747 & $77 \%$ & 42 \\
\hline 15224110146 & $59 \%$ & 42 \\
\hline 15120100409 & $39 \%$ & 41 \\
\hline 14722060926 & $62 \%$ & 38 \\
\hline 15130183944 & $61 \%$ & 33 \\
\hline 15621053651 & $30 \%$ & 33 \\
\hline 14506202425 & $18 \%$ & 30 \\
\hline 15602034535 & $15 \%$ & 26 \\
\hline 14818065039 & $51 \%$ & 18 \\
\hline 15430121514 & $32 \%$ & 14 \\
\hline
\end{tabular}

TABLE 1: Potential Donors with Corresponding KDPI and Age

Potential donors are listed in descending order by age. 
During the data collection time period, the LOPA transplanted 483 kidneys. The additional 25 possible IDSKD would have accounted for an increase of $5.17 \%$ kidneys transplanted. During this same collection period, 23,820 kidneys were transplanted nationally. A 5\% increase nationally could have yielded an estimated 1,191 live-saving transplantable kidneys.

The patient population on whom this study was focused were referred to the LOPA during their most current hospital admittance. Average times from admittance to extubation ranged from 10 hours to 690 hours. The average time from admittance to extubation was 140.8 hours, or 5.8 days. There was no correlation between the length of time to extubation and an increase in a patient's KDPI. Extubation time for case $\# 14506202425$ was not readily available, therefore it was not included in the average calculation.

\section{Discussion}

At this time, the LOPA is formulating an implementation strategy for IDSKD. The most likely scenario for implementation will require a trial period at a local transplant facility. The LOPA would initially evaluate a patient's suitability for donation after circulatory death. If the patient does not meet the criteria, they would then be evaluated for IDSKD. By trialing IDSKD in a local transplant facility, it would allow greater control of the possible variables involved until the process is perfected. Educating and preparing the hospital staff would be considered paramount during this trial period.

As preparation continues, the OPO industry is faced with a primary issue. The United Network for Organ Sharing (UNOS) currently does not recognize imminent death single kidney donation. Therefore, there are no policies in place governing recovery and allocation of recovered kidneys through this process. Additional communication and process formalization with UNOS will be required to move forward in the future.

IDSKD will undoubtedly bring in ethical questions. An anticipated concern is hastening the death of the donor due to the removal of a kidney. According to the National Kidney Foundation, "Living donation does not change life expectancy, and does not appear to increase the risk of kidney failure. In general, most people with a single normal kidney have few or no problem..." [2]. While IDSKD is not technically considered living donation under its formal definition, the risks involved in the two processes have their similarities.

We realize limitations may exist by using the Kidney Donor Profile Index. Primarily, the fact that it does not calculate how well a kidney will actually function once transplanted or just how well it might outperform other kidneys transplanted based on historical data. Fortunately, our goal was not to determine the functionality of the kidneys once transplanted. The goal was to identify a group of patients who, based on criteria provided by our medical director, would possibly be considered suitable for donation. Using the KDPI allowed us to take into account many different patient demographics and provided a metric on which we would be able to filter results. Ultimately, each transplant facility would be responsible for determining their own criteria for acceptance of a kidney based on the information provided to them.

Another task that will need to be addressed lies in how OPOs will be judged by governing agencies, primarily the federal government. Currently, OPOs are judged based on metrics that rate how productive and efficiently they are able to maximize organs from a potential donor. IDSKD would have a direct and negative impact on their efficiency rating.

\section{Conclusions}

While it is clearly evident that imminent death single kidney donation will not completely address the entire shortage of kidneys the nation is currently facing, it does have the potential 
to provide more life-saving transplantable kidneys to patients in need. Ultimately, our responsibility as the organ procurement and transplant community is to ensure that those in need of a transplant are provided with said opportunity. We have a responsibility to be innovative in pursuing all legal and ethical options to ensure that no person dies while waiting for a transplant.

\section{Additional Information}

\section{Disclosures}

Human subjects: All authors have confirmed that this study did not involve human participants or tissue. Animal subjects: All authors have confirmed that this study did not involve animal subjects or tissue. Conflicts of interest: In compliance with the ICMJE uniform disclosure form, all authors declare the following: Payment/services info: All authors have declared that no financial support was received from any organization for the submitted work. Financial relationships: All authors have declared that they have no financial relationships at present or within the previous three years with any organizations that might have an interest in the submitted work. Other relationships: All authors have declared that there are no other relationships or activities that could appear to have influenced the submitted work.

\section{Acknowledgements}

Thank you Michelle Cavett and Kelly Ranum for you continued support and guidance during this process. Thank you also to Dr. Cohen, LOPA Medical Director, for overseeing the development of this project.

\section{References}

1. Kidney Donor Profile Index (KDPI) Guide for Clinicians . Accessed: July 26, 2016: http://optn.transplant.hrsa.gov/resources/guidance/kidney-donor-profile-index-kdpi-guidefor-clinicians/.

2. What to Expect After Donation. (2016). Accessed: July 26, 2016: http://www.kidney.org/transplantation/livingdonors/what-expect-after-donation. 